\title{
Cardiac Troponin I Levels in Children with Acute Severe Asthma Treated with IV Salbutamol
}

\author{
Kulkarni $\mathrm{H}^{3 *}$, Akwei $\mathrm{S}^{3}$, Luyt DK${ }^{3}$, Gaillard EA ${ }^{1,3}$, Mulla $\mathrm{H}^{2}$ and Pandya $\mathrm{HC}^{1,3}$ \\ ${ }^{1}$ Department of Infection, Immunity and Inflammation, University of Leicester, Leicester, UK \\ ${ }^{2}$ Department of Pharmacy, Glenfield Hospital, Leicester, UK \\ ${ }^{3}$ Department of Paediatric Allergy and Respiratory Medicine, University Hospitals Leicester NHS Trust, Leicester, UK
}

\begin{abstract}
Background: Severe acute asthma and its treatment may uncommonly result in cardiac complications such as arrhythmias and acute coronary ischaemia.

Methods: We carried out a retrospective audit of 14 children treated for acute severe asthma with intravenous (IV) salbutamol at the Leicester Royal Infirmary between September 2012 and April 2013. Cardiac troponin I levels were measured at the time blood was sampled for monitoring of blood biochemistry.

Results: Cardiac troponin I (CTnl) levels ranged from 20 to 4255 mg/ml; 8 children had elevated CTnl levels (>40mg/ $\mathrm{ml}$ ) on 9 occasions. Neither total $(\mathrm{mg} / \mathrm{kg}$ ) salbutamol (inhaled + IV) or total $(\mathrm{mg} / \mathrm{kg})$ salbutamol IV dose correlated with blood CTnl levels.

Conclusions: In most patients, raised CTnl were not associated with signs or symptoms of cardiac dysfunction. Our findings highlight the need for close cardiac monitoring of children with acute severe asthma on IV salbutamol therapy.
\end{abstract}

Keywords: Status asthmaticus; Salbutamol; Cardiac Troponin I

\section{Introduction}

Acute severe asthma attacks in children are life-threatening emergencies. With appropriate and prompt treatment most children recover uneventfully. When complications occur, they are usually a consequence of disease progression and/or its treatment. Established cardiac complications associated with acute asthma treatment in children include supraventricular tachycardia (SVT) and acute coronary ischaemia [1,2]. More recently, two groups have reported elevated cardiac troponin I (CTnI) levels in children treated for acute asthma $[3,4]$. In this case series, the raised levels of CTnI were not generally accompanied by overt symptoms or signs of coronary ischaemia. Following an index case of a child with acute severe asthma who developed acute coronary ischaemia in association with elevated CTnI levels and IV salbutamol therapy, our local policy has been to screen/monitor patients on continuous IV salbutamol for cardiac ischaemia using blood CTnI levels. Here, we report our observations of CTnI levels in children treated with intravenous (IV) salbutamol for acute severe asthma.

\section{Methods}

This is a retrospective audit of routine clinical practice. The case mix comprises of children treated for acute severe asthma with IV salbutamol at the Leicester Royal Infirmary between September 2012 and April 2013. All children with severe asthma are managed in adjoining high dependency care or intensive care units. Patients with severe asthma were identified using ward admission databases. The primary source of patient data were case notes. All patients were managed by the on-call clinical team according to local and / or national asthma treatment guidelines and based on their clinical status.

As per our local policy, children with severe asthma were initially treated with inhaled bronchodilators (salbutamol, ipratropium bromide), oxygen and systemic corticosteroids. In our institution, IV salbutamol is second line therapy. Other agents such as IV aminophylline, IV magnesium and IV adrenaline are third line therapy. The 'loading' and 'continuous' doses for IV salbutamol are $15 \mathrm{mcg} / \mathrm{kg}$ over $10 \mathrm{minutes}$ and $1-5 \mathrm{mcg} / \mathrm{kg} /$ minute respectively. Drugs doses were calculated using estimated or measured weight. Patients receiving continuous IV salbutamol for more than 12 hours had CTnI levels performed at the time blood was sampled for monitoring of blood biochemistry.

Clinical details about patients including drug doses were obtained by analysis of HDU and ICU notes and charts and recorded onto GraphPad Prism V6 spread-sheets. In our hospital, blood CTnI levels $>40 \mathrm{ng} / \mathrm{ml}$ or a rise in blood CTnI $>20 \mathrm{ng} / \mathrm{ml}$ is considered abnormal and consistent with acute coronary ischemia. Clinical information was obtained by three of the authors (SS, HK and HP) analysing the notes together. Pearson's coefficient correlation was calculated using GraphPad Prism V6.

\section{Results}

Fourteen children received continuous IV salbutamol for management of acute severe asthma between September 2012 and April 2013 on 15 separate occasions (1 child received continuous IV salbutamol during two separate admissions). In addition to inhaled salbutamol and IV salbutamol, 4 children received a single bolus dose of IV magnesium. One child was transiently treated with IV aminophylline and also received a bolus of IV Magnesium and a bolus of IV adrenaline.

The mean age (range) and weight (range) of children was 4.75 (1.5-11) years and 22.1 (10-53.5) Kg respectively. All but one child had one or previous episodes of wheezing prior to admission and had inhaled salbutamol therapy at home. Nine children were on inhaled corticosteroid therapy as well as inhaled salbutamol; 4 had other features of atopy (eczema, rhinitis or food allergy). Only 1 child was born

*Corresponding author: Hemant Kulkarni, Consultant in Paediatric Respiratory Medicine, University Hospitals of Leicester NHS trust, Leicester, LE1 5WW, UK, Tel: +44(0)116 258 6694; E-mail: hemant.kulkarni@uhl-tr.nhs.uk

Received October 07, 2015; Accepted December 11, 2015; Published December 18,2015

Citation: Kulkarni H, Akwei S, Luyt DK, Gaillard EA, Mulla H, et al (2015) Cardiac Troponin I Levels in Children with Acute Severe Asthma Treated with IV Salbutamol . Lung Dis Treat 1: 102. doi:10.4172/2472-1018.1000102

Copyright: ( 2015 Kulkarni $\mathrm{H}$, et al. This is an open-access article distributed under the terms of the Creative Commons Attribution License, which permits unrestricted use, distribution, and reproduction in any medium, provided the original author and source are credited. 
premature ( 34 weeks). The heart rate of children on admission ranged from 119-176 beats per minute (bpm). The lowest oxygen saturations on admission ranged from $80-91 \%$. Chest radiographs were reported as being consistent with acute asthma ( $\mathrm{N}=12$ occasions) or consistent with a lower respiratory tract infection ( $\mathrm{N}=3$ occasions). No child had a history of cardiac disease. During therapy with IV salbutamol peak heart rate ranged from $175-210 \mathrm{bpm}$. One child required mechanical support. This patient also developed ECG changes and elevated levels of $\mathrm{CTnI}$ and creatine kinase (CK-MB) in blood consistent with severe myocardial ischemia. All children had normal serum creatinine levels and liver functions tests during their hospital admission and recovered fully from their asthma exacerbation. Cardiac troponin I levels ranged from 20 to 4255; 8 patients had elevated CTnI levels $(>40 \mathrm{mg} / \mathrm{ml})$ on 9 occasions. All patients with elevated CTnI levels during their asthma exacerbation had normal blood CTnI levels $(<20 \mathrm{ng} / \mathrm{ml}) 2$ weeks post discharge from hospital.

Neither total $(\mathrm{mg} / \mathrm{kg})$ salbutamol (inhaled + IV) or total $(\mathrm{mg} / \mathrm{kg})$ salbutamol IV dose correlated with blood CTnI levels (Figure 1a, $\mathrm{R}^{2}$ $=0.009$ and 0.005 respectively, Pearson product-moment correlation coefficient, PPMCC). There is a weak correlation between total $(\mathrm{mg} / \mathrm{kg})$ salbutamol IV dose and blood CTnI $\left(\mathrm{R}^{2}=0.28\right.$, PPMCC) if the child with acute coronary syndrome is excluded from analysis. There was a tendency for CTnI levels to correlate with patient age (Figure $1 \mathrm{~b}, \mathrm{R}^{2}=$ 0.33, Pearson correlation coefficient).

\section{Discussion}

The primary finding of this report is that blood cardiac troponin I (CTnI) levels are elevated in most children experiencing an acute severe exacerbation of asthma and requiring continuous IV salbutamol. Since $\mathrm{CTnI}$ is an intracellular protein expressed only by myocardial cells, our observations suggest that children with acute severe asthma are at risk of myocardial injury.

In children, elevated levels of CTnI are most commonly associated with overt evidence of cardiac dysfunction, for example, following cardiac surgery or a coronary hypoxia-ischaemia episode. In this report most children experiencing a severe asthma attack had outwardly normal cardiac function. Electrocardiographic changes consistent with myocardial ischemia were observed only in one child who had the highest CTnI level. None of the group developed cardiac rhythm disturbances. Thus, in most children with severe asthma and an elevated CTnI level, there was little overt evidence of cardiac dysfunction.

Salbutamol and corticosteroids were the only drugs used in all children. There is no data to suggest that systemic corticosteroid therapy at doses used in childhood asthma cause acute myocardial damage. In contrast, salbutamol therapy has been associated with myocardial necrosis in adults treated for severe asthma [2]. It has also been shown to induce dose-dependent myocardial necrosis in healthy mice [2]. While a dose-dependent effect would implicate salbutamol in elevating CTnI levels in our cohort, we did not find any correlation between weight-adjusted salbutamol doses and blood CTnI levels (Figure 1a). However, on re-performing this analysis after exclusion of the patient with overt coronary ischaemia who received bolus IV adrenaline, we noted a weak correlation between weight-adjusted salbutamol doses and blood CTnI levels. We felt justified to exclude this case in a reanalysis as factors such as IV adrenaline therapy and decreased cardiac output could have also contributed to their overall CTnI level. An additional or alternative explanation for a weak correlation between CTnl levels and salbutamol therapy is that dose-response relationship is 'sigmoid' and that all children in this case series report were in the plateau phase of this relationship.
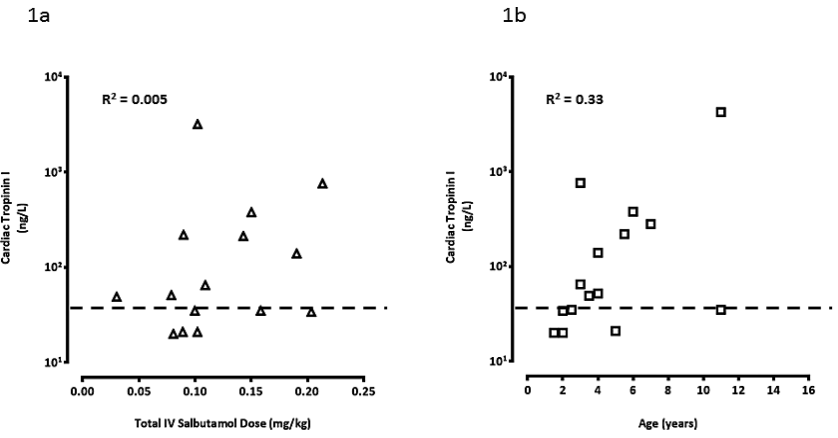

Figure 1: Cardiac tropinin I levels plotted against dose of intravenous salbutamol and age. Total IV salbutamol dose did not correlate with blood CTnl levels. (1a): There was a tendency for CTnl levels to correlate with patient age. (1b): Dotted line represents $C T n l=40 \mathrm{ng} / \mathrm{ml} ; \mathrm{N}=14$ patients; $\mathrm{R}^{2}$ $=$ Pearson product-moment correlation coefficient.

The tendency of CTnI levels to correlate with increasing age (Figure 1b) suggests that myocyte sensitivity to salbutamol is age-dependent. One potential explanation to account for this association could lie in the relationship between heart rate and coronary perfusion during diastole. Since baseline and maximal heart rates vary inversely with age, it seems reasonable to speculate that cardiac myocytes of young children tolerate periods of sustained tachycardia, as might occur with salbutamol therapy, better than cardiac myocytes of older children [5].

Whether the raised CTnIlevels in this case auditare a manifestation of asthma severity and/or salbutamol therapy is unknown. Kalyanaraman et al. have previously noted that 3 of 20 children with severe acute asthma had raised CTnI levels while receiving inhaled salbutamol only. However, after commencing IV terbutaline, 9 children had a rise in CTnI levels [4]. One patient in this report developed ECG changes diagnostic of myocardial injury. As in our case, this patient had very high CTnI levels and their ECG normalized on discontinuation of IV bronchodilator therapy. While this data suggest both disease severity and total doses of $\beta$-agonists contribute to changes in blood CTnI, Kalyanaraman et al. found no association between asthma severity scores and elevated blood CTnI levels.

Clearly, further studies are needed to define the mechanism of raised CTnI in children with asthma. For example, to properly investigate salbutamol's potential dose-toxicity relationship will require a prospective study of a range of doses and multiple, repeated observations (CTnl levels) over the dosing interval. In the meantime, our findings highlight the need for close monitoring of children with acute severe asthma on IV Salbutamol therapy.

\section{References}

1. Travers AH, Milan SJ, Jones AP, Camargo CA Jr, Rowe BH (2012) Addition of intravenous beta(2)-agonists to inhaled beta(2)-agonists for acute asthma. Cochrane Database Syst Rev 12: CD010179.

2. Libretto SE (1994) A review of the toxicology of salbutamol (albuterol). Arch Toxicol 68: 213-216

3. Bogie AL, Towne D, Luckett PM, Abramo TJ, Wiebe RA (2007) Comparison of intravenous terbutaline versus normal saline in pediatric patients on continuous high-dose nebulized albuterol for status asthmaticus. Pediatr Emerg Care 23 355-361.

4. Kalyanaraman M, Bhalala U, Leoncio M (2011) Serial cardiac troponin concentrations as marker of cardiac toxicity in children with status asthmaticus treated with intravenous terbutaline. Pediatr Emerg Care 27: 933-936.

5. Bache RJ, Cobb FR (1977) Effect of maximal coronary vasodilation on transmural myocardial perfusion during tachycardia in the awake dog. Circ Res 41: 648-653. 